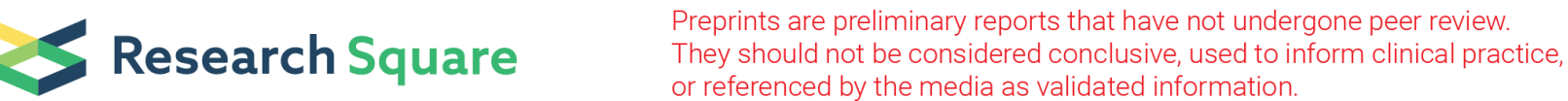

\section{Good visual rehabilitation in a patient without traumatic cataract surgery after removal of a ferrous intravitreal foreign body by external}

magnetic extraction through a pars plana incision: $A$ case report

\section{Zhitao Su}

Eye Center, Second Affiliated Hospital, School of Medicine, Zhejiang University

\section{Panpan Ye}

Eye Center, Second Affiliated Hospital, School of Medicine, Zhejiang University

Jijian Lin

Eye Center, Second Affiliated Hospital, School of Medicine, Zhejiang University

Xiaodan Huang

Eye Center, Second Affiliated Hospital, School of Medicine, Zhejiang University

Xiaoyun Fang ( $\nabla$ xiaoyunfang@zju.edu.cn )

Zhejiang University School of Medicine Second Affiliated Hospital https://orcid.org/0000-0001-50378675

\section{Case report}

Keywords: Intravitreal foreign body, Traumatic cataract, External approach, Case report

Posted Date: May 3rd, 2019

DOI: https://doi.org/10.21203/rs.2.319/v3

License: (c) (i) This work is licensed under a Creative Commons Attribution 4.0 International License.

Read Full License 


\section{Abstract}

Background: There is an increasing trend toward performing pars plana vitrectomy and simultaneous cataract extraction in the management of patients with intraocular foreign bodies and traumatic cataracts. Herein, we describe a case of good visual rehabilitation in a patient without traumatic cataract surgery after removal of a ferrous intravitreal foreign body (IVFB) using an external approach. Case presentation: A 44-year-old man presented to our ophthalmology department because of decreased visual acuity after a penetrating trauma to the left eye 2 days earlier. Best-corrected visual acuity (BCVA) was 20/200. An examination revealed a midperipheral self-sealing corneal penetrating wound at the 5 o'clock position, in addition to a mild inflammatory reaction in the anterior chamber. Pupil dilation revealed peripheral anterior and posterior capsular violations, with subcapsular opacity involving the visual axis. A small metallic-like foreign body suspended in the vitreous was confirmed by B-scan ultrasonography and orbital computed tomography. Retinal injury and vitreous hemorrhage were excluded by B-scan ultrasonography and binocular indirect ophthalmoscopy. This IVFB was successfully removed by external magnetic extraction through a pars plana incision. At the 2-week follow-up, the traumatic cataract was partially resolved, and BCVA improved to 20/125. At the 3-month follow-up, the traumatic cataract was mostly resolved, the visual axis was clear under a normal pupil, and BCVA improved to 20/20. Peripheral localized lens opacity was foun

\section{Background}

Among patients with a posterior segment intraocular foreign body (IOFB) and a traumatic cataract, visual rehabilitation poses a unique challenge to ophthalmic surgeons. There is an increasing trend toward performing pars plana vitrectomy (PPV) and simultaneous cataract extraction in the management of these patients [1-3]. However, previous study reported spontaneous resolution of a traumatic cataract after removal of an intralenticular foreign body and lens preservation remaining localized opacity after IOFB removal by PPV [4-5]. The external approach to the removal of a metallic IOFB remains to be a viable treatment option in select cases [6]. Herein, we describe a case of traumatic cataract caused by a small ferrous intravitreal foreign body (IVFB), in which good visual rehabilitation was achieved without traumatic cataract surgery after removal of the IVFB through a pars plana incision using an external magnet.

\section{Case Presentation}

A 44-year-old healthy man was referred to our ophthalmology department because of decreased visual acuity in his left eye for 2 days. The patient had suffered a penetrating trauma to his left eye while hammering metal without safety glasses 2 days before. On presentation, the best corrected visual acuity (BCVA) was 20/20 in his right eye and 20/200 in his left eye. The intraocular pressure (IOP) was 16 $\mathrm{mmHg}$ in his right eye and $13 \mathrm{mmHg}$ in his left eye. A slit lamp examination revealed a 2-mm self-sealing midperipheral corneal penetrating wound at the 5 o'clock position in the patient's left eye. There was a mild inflammatory response in the anterior chamber. Pupil dilation revealed peripheral anterior and 
posterior capsular violations, with subcapsular opacity involving the visual axis (Figs. 1A-C). A scanning laser ophthalmoscopic image showed a dense shadow in the middle and inferotemporal visual field that was caused by the traumatic cataract (Fig. 1D). A small metallic-like foreign body suspended in the vitreous at the 5 o'clock position was confirmed by B-scan ultrasonography and orbital computed tomography (Figs. 1E, F). Retinal injury and vitreous hemorrhage were excluded by B-scan ultrasonography and binocular indirect ophthalmoscopy. This IVFB, $1.0 \mathrm{~mm}$ in width and $2.5 \mathrm{~mm}$ in length, was successfully removed by external magnetic extraction through a pars plana incision. Briefly, the pars plana at the 5 o'clock position was exposed by opening the bulbar conjunctiva. Preplacing a sclerotomy suture was performed to allow quick closure once the foreign body was removed. A $3.0 \mathrm{~mm}$ sclerotomy, $4.0 \mathrm{~mm}$ from the corneal limbus, was made. The choroid was diathermized and incised. A rare earth magnet was placed at the sclerotomy site, and the foreign body was removed. The vitreous attached to the foreign body was cut off before the foreign body left the sclera. The sclerotomy and the open conjunctiva were closed by an 8-0 absorbable polyglactin 910 suture (Johnson \& Johnson, W9560). Postoperatively, the patient received $0.5 \%$ levofloxacin eye drops and $1 \%$ prednisolone acetate eye drops 4 times a day for 1 week, $1 \%$ pranoprofen eye drops 4 times a day for 4 weeks, and $0.5 \mathrm{~g}$ levofloxacin tablet a day for 4 days. The patient was followed up 1 day, 3 days, 2 weeks, 3 months, 6 months, and 12 months after the surgery. If necessary, traumatic cataract removal by phacoemulsification and intraocular lens implantation were planned.

At the follow-up of 2 weeks after the surgery, the BCVA had improved to 20/125, the traumatic cataract had partially resolved (Figs. $2 \mathrm{~A}, \mathrm{~B}$ ), the range and density of the shadow reduced (Fig. 2C), and there were no signs of endophthalmitis or retinal detachment. At the follow-up of 3 months after the surgery, the BCVA was 20/20. A slit lamp examination revealed a midperipheral corneal scar at the 5 o'clock position, with no anterior chamber inflammation. The visual axis was clear under a normal pupil. Pupil dilation revealed peripheral localized lens opacity, without visual axis involvement (Figs. 2 D, E). A scanning laser ophthalmoscopic image showed a slight temporal shadow (Fig. 2F). A fundus examination revealed no obvious abnormality.

\section{Discussion And Conclusions}

The prognosis of traumatic eye injuries associated with IOFBs varies greatly depending on a number of factors, which include the time between trauma and IOFB extraction, initial visual acuity, entrance wound location, nature of IOFB, location of IOFB, preoperative retinal detachment, presence of intraocular hemorrhage, presence of endophthalmitis, and primary surgical repair combined with IOFB removal and the occurrence of postoperative complications [7]. PPV is the most common surgical approach for the removal of a posterior segment IOFB, as it provides direct viewing and controlled surgery [8-9]. The aim of the treatment is to restore ocular integrity and to obtain a good visual outcome. However, in selected patients with small ferrous foreign bodies positioned in the vitreous and no retinal injury, a good visual outcome can be achieved by removal of the IVFB by external magnetic extraction through a pars plana incision [6]. 
We described the case of a 44-years-old healthy man with a traumatic cataract involving the visual axis caused by a small metallic-like IVFB in his left eye. The IVFB was removed by external magnetic extraction through a pars plana incision. Spontaneous resolution of the traumatic cataract was observed. At the last follow-up 12 months after the removal of the IVFB, BCVA improved to 20/20, stable localized lens opaciy, not involving the visual axis, was found, and a fundus examination showed no obvious abnormality.

Lenticular injury as a result of an IOFB may occur if the foreign body passes through the lens [10]. Removal of an IOFB in the presence of a traumatic cataract and associated retinal pathology is difficult. Under such circumstances, cataract extraction is often necessary to enable clear visualization of the posterior segment. Important advances in microsurgical and vitreoretinal instrumentation and techniques (e.g., wide angle viewing systems, high-speed cutters, and improved intraocular instrumentation) have improved the outcomes of these types of ocular injuries.

In cases of clear lens damage and extensive cortical material in the anterior chamber, which may cause increased IOP or a severe inflammatory reaction, lens removal is performed. However, a minor injury to the lens may result in localized nonprogressive lens opacity that does not require surgery. In these cases, epithelial cells may regenerate at the site of the injury and restore capsular continuity, thus limiting the free passage of ions and fluid that can result in progressive cataract formation [11].

In the present case, a small metallic-like IVFB was identified by B-scan ultrasonography and orbital computed tomography, and no signs of endophthalmitis, vitreous hemorrhage, or retinal injury were found. In such cases, removal of the IVFB using an external approach is an easy and a viable treatment option. To allow clear visualization of the posterior segment for the removal of this IVFB by PPV, extraction of the traumatic cataract, which interfered with the visual axis, was necessary. Removal of the posterior hyaloid, an important surgical goal, was difficult in this relatively young patient. There was also a potential drop of this IVFB on the macula.

In the absence of lens material in the anterior chamber in the presence of a traumatic cataract, some literature states that it is better to treat the eye with topical steroids to control inflammation firstly. In the present case, a slit lamp examination revealed a mild inflammatory reaction in the anterior chamber and no cortical material in the chamber. The patient's IOP was normal. Small peripheral anterior and posterior capsular violations were observed. Although the traumatic cataract interfered with the visual axis, considering the size and location of the capsular violations and the mild nature of the inflammatory reaction, in addition to the potential possibility of restoration of capsular continuity and spontaneous resolution of the traumatic cataract, the patient was treated with topical antibiotic and steroids to control the inflammation after the removal of the IVFB, and lens extraction was deferred. During the first 3 months follow-up after removal of the IVFB by external approach, the traumatic cataract was mostly resolved. The visual axis was clear under normal pupil and BCVA improved to 20/20. After pupil dilation, temporal localized lens opacity was found. There were no postoperative complications, such as secondary glaucoma or retinal detachment. At the last follow-up of 12 months after the surgery, localized 
lens opacity remained stable and BCVA was 20/20. A fundus examination revealed no obvious abnormality.

Here we report a case of good visual rehabilitation without cataract and vitrectomy surgery in a patient with a penetrating eye injury, accompanied by an IVFB and a traumatic cataract. This case emphasizes the need for clinical education in terms of the management of an IVFB. When determining the need for a surgical intervention or conservative management in such cases, patient-related factors, the size and location of the IOFB, the location and extent of lenticular involvement, and associated injuries should be considered to obtain a good visual outcome.

\section{Abbreviations}

BCVA: Best corrected visual acuity; IOFB: intraocular foreign body; IOP: intraocular pressure; IVFB: intravitreal foreign body; PPV: pars plana vitrectomy.

\section{Declarations}

Ethics approval and consent to participate: This study has been performed in accordance with the Declaration of Helsinki and was approved by the Ethics Committee of the Second Affiliated Hospital, School of Medicine, Zhejiang University.

Consent for publication: Written informed consent was obtained from the patient.

Availability of data and materials: The datasets used and analyzed during the current study are available from corresponding author on reasonable request.

Competing interests: The authors declare that they have no competing interests

Funding: This study was supported by Natural Science Foundation of Zhejiang Province LY15H120001 and National Natural Science Foundation of China (Grant No. 81370019 and 81870624). The funding bodies did not have a role in the study design, data collection, data analysis, interpretation of data, writing the manuscript, the critical revision and approval of submission of the current study.

Authors' contributions: ZTS collected the data and drafted the manuscript. PPY and JLL collected the data. XDH participated in the diagnosis and treatment of the patient. XYF guided the study and revised the manuscript. All authors have read and approved the final manuscript.

\section{Acknowledgements: None References}

1. Dhoble P, Khodifad A. Combined cataract extraction with pars plana vitrectomy and metallic intraocular foreign body removal through sclerocorneal tunnel using a novel "magnet handshake" technique. Asia Pac J Ophthalmol (Phila). 2018;7(2):114-118. 
2. Batman C, Cekic O, Totan Y, Ozkan SS, Zilelioglu O. Combined phacoemulsification, vitrectomy, foreignbody extraction, and intraocular lens implantation. J Cataract Refract Surg. 2000: 26(2):254-259.

3. Vatavuk Z, Pentz A. Combined clear cornea phacoemulsification, vitrectomy, foreign body extraction, and intraocular lens implantation. Croat Med J. 2004;45(3):295-298.

4. Rofagha S, Day S, Winn BJ, Ou JI, Bhisitkul RB, Chiu CS. Spontaneous resolution of a traumatic cataract caused by an intralenticular foreign body. J Cataract Refract Surg. 2008; 34(6):1033-1035.

5. Pieramici DJ, Capone AJ, Rubsamen PE, Roseman RL. Lens preservation after intraocular foreign body injuries. Ophthalmology. 1996: 103(10):1563-1567.

6. Chow DR, Garretson BR, Kuczynski B, Williams GA, Margherio R, Cox MS, et al. External versus internal approach to the removal of metallic intraocular foreign bodies. Retina. 2000; 20(4):364-369.

7. Oztas Z, Nalcaci S, Afrashi F, Erakgun T, Mentes J, Degirmenci C, et al. Posterior segment intraocular foreign bodies: the effect of weight and size, early versus late vitrectomy and outcomes. Ulus Travma Acil Cerrahi Derg. 2015;21(6):496-502.

8. Yuksel K, Celik U, Alagoz C, Dundar H, Celik B, Yazici AT. 23 gauge pars plana vitrectomy for the removal of retained intraocular foreign bodies. BMC Ophthalmol. 2015; 15:75.

9. Kuhn F, Morris R. Posterior segment intraocular foreign bodies: management in the vitrectomy era. Ophthalmology. 2000; 107(5):821-822.

10. Moisseiev J, Segev F, Harizman N, Arazi T, Rotenstreich Y, Assia El. Primary cataract extraction and intraocular lens implantation in penetrating ocular trauma. Ophthalmology. 2001; 108(6):1099-1103.

11. Fagerholm PP, Philipson BT. Human traumatic cataract. A quantitative microradiographic and electron microscopic study. Acta Ophthalmol (Copenh). 1979;57(1):20-32.

12. Moisseiev J, Segev F, Harizman N, Arazi T, Rotenstreich Y, Assia El. Primary cataract extraction and intraocular lens implantation in penetrating ocular trauma. Ophthalmology. 2001; 108(6):1099-1103.

\section{Figures}



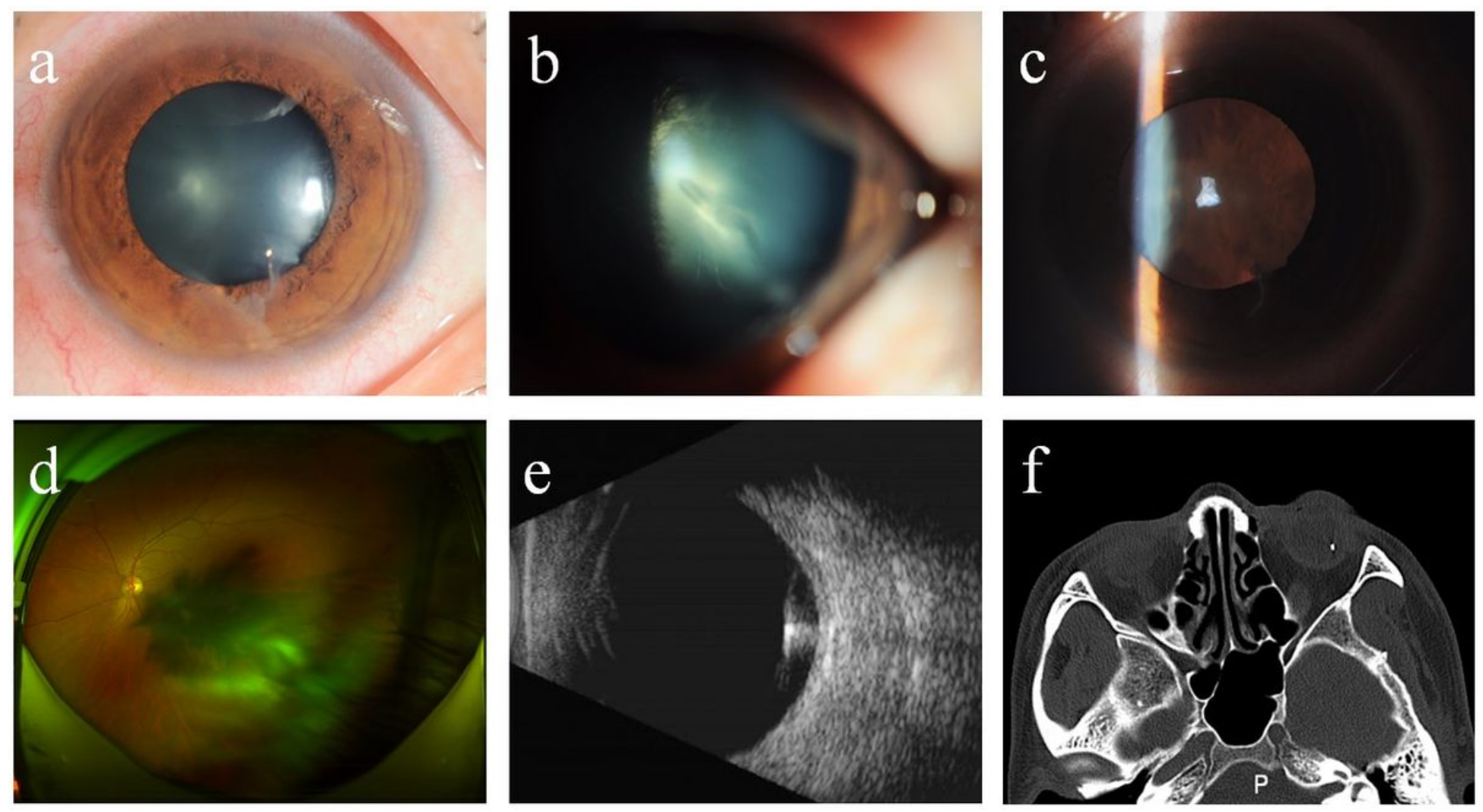

Figure 1

An anterior segment photograph revealed a 2-mm self-sealing midperipheral corneal penetrating wound at the 5 o'clock position, with anterior capsular violation (a), posterior capsular violation (b), and significant subcapsular opacity involving the visual axis (c). A scanning laser ophthalmoscopic image showed a dense shadow caused by the traumatic cataract (d). A small metallic-like foreign body suspended in the vitreous without retinal injury was confirmed by B-scan ultrasonography (e) and orbital computed tomography (f). 

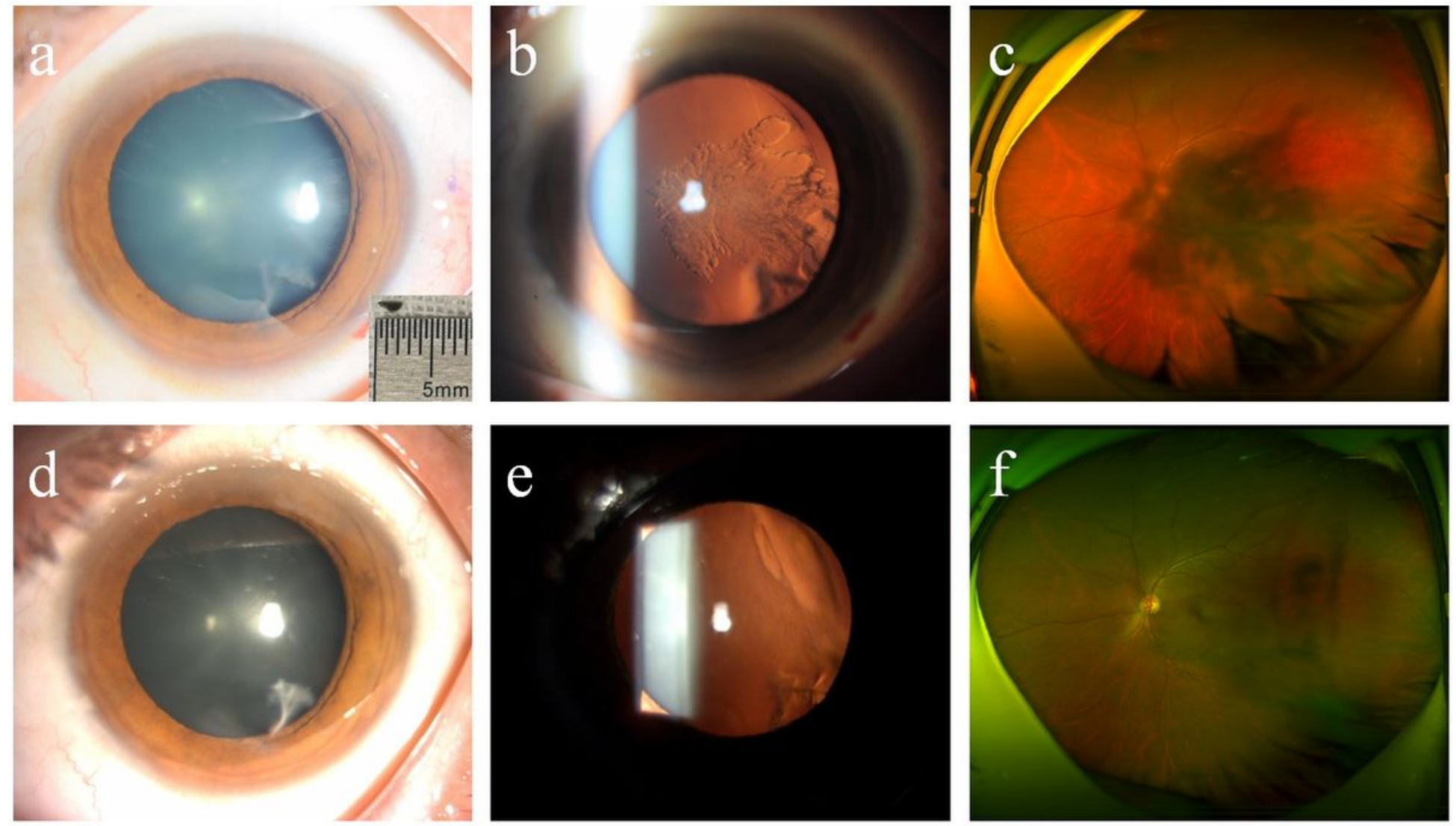

\section{Figure 2}

A $1 \times 2.5 \mathrm{~mm}$ sized metallic foreign body was successfully removed by external magnetic extraction through a pars plana incision (a). At the follow-up of 2 weeks after the surgery, the traumatic cataract had partially resolved $(a, b)$, the range and density of the shadow reduced (c). At the follow-up of 3 months after the surgery, the traumatic cataract had mostly resolved, a midperipheral corneal scar (d) and peripheral localized lens opacity (e) were observed. A scanning laser ophthalmoscopic image showed a slight temporal shadow (f).

\section{Supplementary Files}

This is a list of supplementary files associated with this preprint. Click to download.

- CAREchecklist.pdf 